\section{Planar polarization of the atypical myosin Dachs orients cell divisions in Drosophila}

\author{
Yanlan Mao, ${ }^{1,2,3}$ Alexander L. Tournier, ${ }^{1,2}$ \\ Paul A. Bates, ${ }^{2}$ Jonathan E. Gale, ${ }^{4}$ \\ Nicolas Tapon, ${ }^{1,6}$ and Barry J. Thompson ${ }^{3,5}$ \\ ${ }^{1}$ Apoptosis and Proliferation Control Laboratory, Cancer \\ Research UK, London Research Institute, London WC2A 3PX, \\ United Kingdom; ${ }^{2}$ Biomolecular Modeling Laboratory, Cancer \\ Research UK, London Research Institute, London WC2A 3PX, \\ United Kingdom; ${ }^{3}$ Epithelial Biology Laboratory, Cancer \\ Research UK, London Research Institute, London WC2A 3PX, \\ United Kingdom; ${ }^{4}$ University College London Ear Institute, \\ University College London, London WC1X 8EE, \\ United Kingdom
}

Tissues can grow in a particular direction by controlling the orientation of cell divisions. This phenomenon is evident in the developing Drosophila wing epithelium, where the tissue becomes elongated along the proximal-distal axis. We show that orientation of cell divisions in the wing requires planar polarization of an atypical myosin, Dachs. Our evidence suggests that Dachs constricts cell-cell junctions to alter the geometry of cell shapes at the apical surface, and that cell shape then determines the orientation of the mitotic spindle. Using a computational model of a growing epithelium, we show that polarized cell tension is sufficient to orient cell shapes, cell divisions, and tissue growth. Planar polarization of Dachs is ultimately oriented by long-range gradients emanating from compartment boundaries, and is therefore a mechanism linking these gradients with the control of tissue shape.

Supplemental material is available for this article.

Received March 15, 2010; revised version accepted November 29, 2010.

Tissues grow in a precisely controlled way to produce adult structures of the correct size and shape. While many molecular pathways controlling tissue size are known (Lawrence and Struhl 1996; Hafen 2004; Pan 2007), little is known about the control of tissue shape. The orientation of cell divisions is an important influence on the shape of Drosophila appendages, such as the wing (Lawrence and Struhl 1996; Baena-Lopez et al. 2005; Lecuit and Le Goff 2007). The wing grows preferentially along its proximal-distal (P-D) axis, and the orientation of cell divisions in this axis is manifest in the elongated shapes of marked clones of cells in the developing wing imaginal disc (Baena-Lopez et al. 2005). How cell divisions

[Keywords: Drosophila; morphogen; shape; proliferation; growth; tension; computational modeling]

Corresponding authors.

${ }^{5}$ E-MAIL barry.thompson@cancer.org.uk; FAX 44-20-7269-3581.

${ }^{6}$ E-MAIL nic.tapon@cancer.org.uk; FAX 44-20-7269-3638.

Article is online at http://www.genesdev.org/cgi/doi/10.1101/gad.610511. are oriented remains largely unknown, but the mechanism must involve orientation of the mitotic spindle, which correlates, albeit imperfectly, with the P-D axis (BaenaLopez et al. 2005).

The Dachsous (Ds) protein is required for control of both wing size and shape (Clark et al. 1995; Baena-Lopez et al. 2005). It has been found that Dachsous is required for the elongation of clones along the $\mathrm{P}-\mathrm{D}$ axis of the wing disc, but how it functions in this process is not yet understood (Baena-Lopez et al. 2005). Much more is known about the role of Dachsous in the control of tissue size. Dachsous is a transmembrane protein that is expressed in a gradient along the $\mathrm{P}-\mathrm{D}$ axis in response to organizing cues emanating from the compartment boundaries, such as the secreted morphogen Dpp (Fig. 1A,B; Clark et al. 1995; Matakatsu and Blair 2004; Simon 2004; Rogulja et al. 2008). An antagonist of Dachsous, Four-jointed, is expressed in an opposing gradient (Mahoney et al. 1991; Cho and Irvine 2004; Matakatsu and Blair 2004; Simon 2004; Rogulja et al. 2008). Dachsous is the ligand for Fat, another transmembrane protein that transduces the Dachsous signal (Mahoney et al. 1991; Cho and Irvine 2004; Matakatsu and Blair 2004; Simon 2004; Cho et al. 2006; Rogulja et al. 2008) via Dachs, an atypical myosin, to the transcriptional activator Yorkie /Cho and Irvine 2004; Bennett and Harvey 2006; Cho et al. 2006; Mao et al. 2006; Silva et al. 2006; Willecke et al. 2006, 2008; Feng and Irvine 2007; Rogulja et al. 2008). Loss of Dachs causes tissue undergrowth (Mao et al. 2006), while loss of Dachsous or Fat has the opposite phenotype: tissue overgrowth (Mahoney et al. 1991; Clark et al. 1995; Cho et al. 2006). In contrast, loss of Dachs, Dachsous, or Fat has similar effects on tissue shape (Mao et al. 2006) - namely, a shortening of the $\mathrm{P}-\mathrm{D}$ axis relative to the anteriorposterior $(\mathrm{A}-\mathrm{P})$ axis-suggesting that these proteins control shape by a different mechanism from the one by which they control tissue size.

\section{Results and Discussion}

Recently, Dachs was found to be localized in a planarpolarized manner along the $\mathrm{P}-\mathrm{D}$ axis in response to the Dachsous gradient (Supplemental Fig. S1; Rogulja et al. 2008). We observed that the localization of Dachs correlates with the orientation of cell divisions and tissue growth in the developing fly wing (Fig. 1C,D; Supplemental Figs. S1, S2). Dachs localizes to the distal side of each cell's apical surface (Fig. 1D; Rogulja et al. 2008), and clones tend to grow preferentially along the P-D axis to form elongated shapes (Fig. 1C). This intriguing correlation led us to speculate that Dachs might mediate the orientation of cell divisions and tissue growth in the fly wing.

To test this hypothesis, we examined the behavior of marked clones of cells in wing discs lacking functional Dachs protein (dachs mutant), and wing discs in which Dachs is abnormally localized around the entire apical cell surface (fat mutant) (Rogulja et al. 2008). The normal elongation of clone shapes along the P-D axis (Fig. 1E) is completely disrupted in either dachs (Fig. 1F) or fat (Fig. 1G) mutant discs, with clones tending to be rounded and misoriented (Fig. 1H). Orientation of mitotic spindles is also disrupted in these mutants (Fig. 1I-K). This failure to 

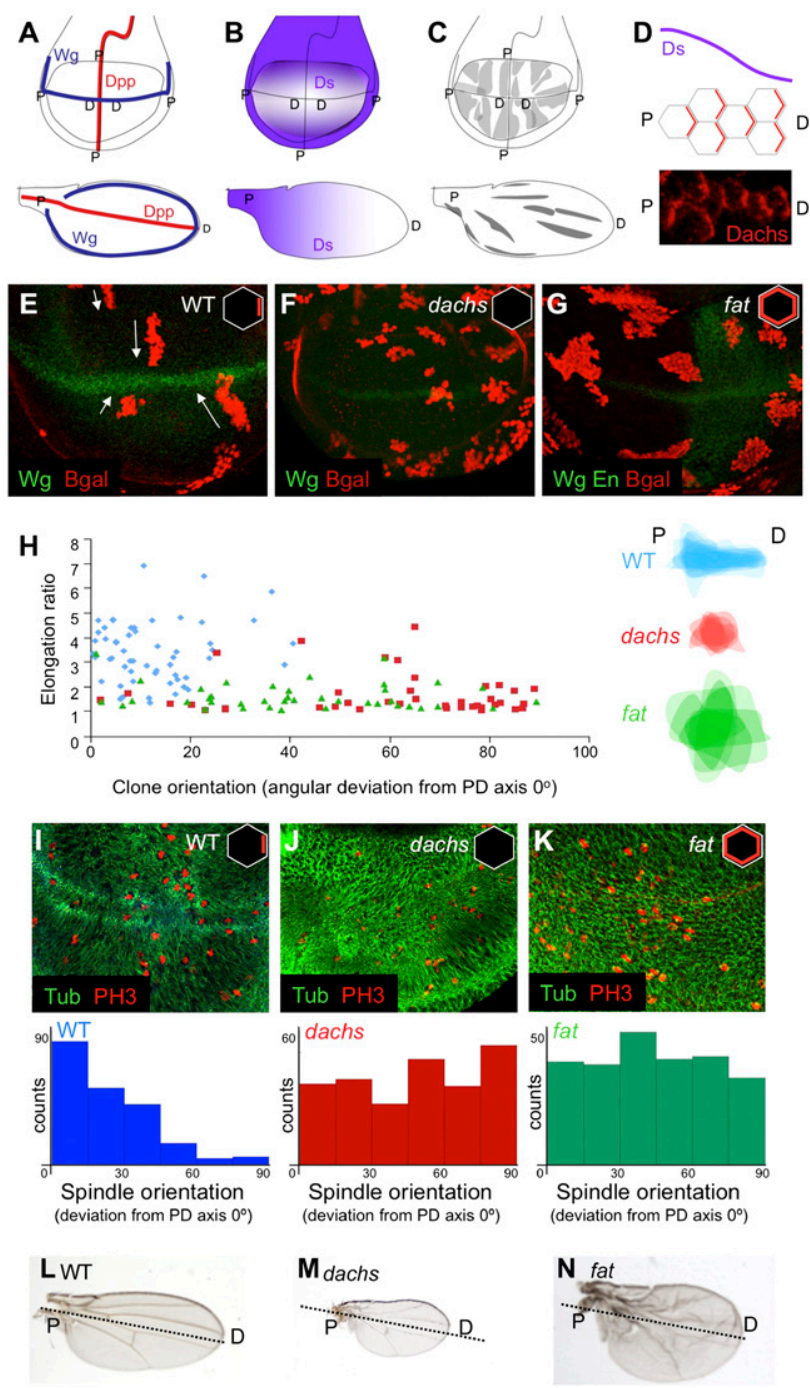

Figure 1. Planar polarization of Dachs is required for orientation of clone and tissue growth. $(A, t o p)$ The Dpp and $\mathrm{Wg}$ morphogens are expressed along the A-P and dorsal-ventral (D-V) compartment boundaries, respectively, in the wing imaginal disc. (Bottom) The corresponding positions in the pupal wing. $(B, t o p)$ Dachsous is expressed in a gradient from proximal (P) to distal (D). (Bottom) The corresponding gradient in the pupal wing. $(C, t o p)$ Clone shapes (gray) in the wing pouch (the future wing) of the wing disc are oriented along the P-D axis. (Bottom) Clone shapes (gray) in the adult wing are also oriented along the P-D axis. $(D)$ The Dachsous (Ds) gradient orients the planar polarization of Dachs (red) along the P-D axis such that Dachs localizes to the distal side of each cell's apical surface. (Bottom) A clone in the wing disc expressing Dachs-V5. $(E-H)$ The shape of clones randomly induced by heat shock and marked by the expression of the lacZ gene (anti-ßgal) was examined in the wing pouch. $(E)$ Clones are oriented along the P-D axis in wild-type discs. $(F)$ Clones fail to orient in dachs mutants. $(G)$ Clones fail to orient in fat mutants. $(H)$ Quantification of E-G (see Supplemental Fig. S6), with overlays of clone shapes shown on the right. Mean clone elongation ratios are as follows: wild-type $=3.4 \pm 1.2(n=55)$, dachs mutant = $1.6 \pm 0.8(n=40, P<0.001)$, fat mutant $=1.6 \pm 0.5(n=41, P<$ $0.001)$. (I-K) Spindle orientations determined by Tub and phosphoHistoneH3 staining were quantified relative to the P-D axis for wildtype $(n=199)(I)$, dachs mutant $(n=236)(J)$, and fat mutant wing discs $(n=235)(K)$. $(L)$ Wild-type wing. $(M)$ dachs mutant wing. $(N)$ fat mutant wing. orient cell divisions in dachs and fat mutants results in abnormally shaped adult wings that are reduced in the $\mathrm{P}-\mathrm{D}$ axis relative to wild-type controls (Fig. 1L-N; Mao et al. 2006). This shape change is evident despite opposite effects of the two mutations on size. These results indicate that planar polarization of Dachs is essential for orienting cell divisions and tissue growth.

We next examined whether reorientation of Dachs is sufficient to reorient cell divisions and tissue growth. We expressed Dachsous with the dpp.Gal4 driver, which is expressed in a gradient along the A-P axis in the anterior compartment (Fig. 2A). This ectopic gradient of Dachsous runs perpendicular to the endogenous gradient and repolarizes Dachs (Fig. 2B,C). As a result, clones are reoriented perpendicular to the $\mathrm{P}-\mathrm{D}$ axis (Fig. 2B,C), as are mitotic spindles (Fig. 2D). In adult wings, the ectopic Dachsous gradient drives elongation of the wing perpendicular to the P-D axis, in the anterior compartment (Fig. 2E-G).

How might Dachs control the orientation of the mitotic spindle? Dachs might directly orient the mitotic spindle by tethering. Alternatively, since Dachs is a myosin, it might indirectly orient the spindle by controlling cell shape. Existing evidence supports the latter view:

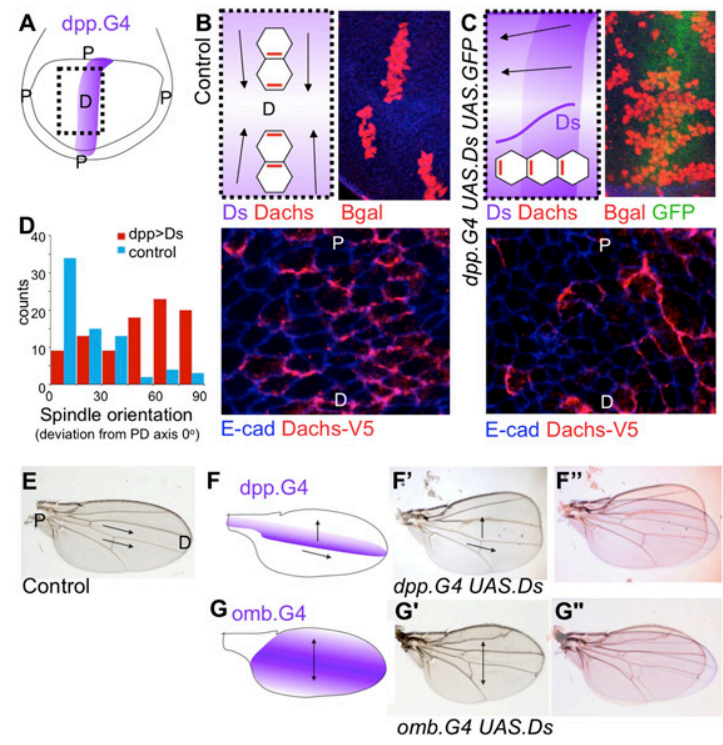

Figure 2. Reorientation of Dachs with a new Dachsous gradient is sufficient to reorient tissue growth. (A) Diagram of the graded expression of dpp.Gal4, which is highest at the A-P compartment boundary and decays into the apical compartment. The dotted line shows the location of the region of interest. $(B, t o p)$ Portion of a wildtype wing pouch showing oriented clones ( $\beta$ gal) along the P-D axis (vertical). A diagram of the endogenous gradient of Dachsous (Ds) in this portion of the wing pouch is shown to the left. (Bottom) Expression of Dachs-V5 using dpp.Gal4 shows polarization in the P-D axis. $(C$, top $)$ Portion of a wing pouch expressing Dachsous (Ds) and GFP with $d p p$.Gal4 driver. Clones (Bgal) orient according to the new Ds gradient. (Bottom) Expression of Dachs-V5 under dpp.Gal4 $U A S$.Ds shows polarization of Dachs along the new Ds gradient. $(D)$ Quantification of spindle orientation in $B(n=70)$ versus $C(n=91)$. $(E)$ Wild-type wing. (F) Pattern of $d p p$.Gal4 in the adult wing. $\left(F^{\prime}\right)$ A dpp.Gal4 UAS.Ds wing grows outward, perpendicular to the P-D axis, in the anterior compartment. $\left(F^{\prime \prime}\right)$ Overlay with a wild-type wing. (G) Pattern of omb.Gal4 in the adult wing. $\left(G^{\prime}\right)$ An omb.Gal4 $U A S$.Ds wing grows outward, perpendicular to the $\mathrm{P}-\mathrm{D}$ axis, in the both compartments. $\left(G^{\prime \prime}\right)$ Overlay with a wild-type wing. 
Mitotic spindles align along the long axis of the cell prior to division in both yeast and mammalian cells (Thery et al. 2007; Vogel et al. 2007). In the case of epithelial cells, spindles are restricted to the plane of the epithelium (Lu et al. 2001) and their orientation may be affected by apical surface geometry. We examined apical cell shape and the orientation of cell division in live wing discs in culture (Supplemental Movie S1) and found that cells divide along their long apical axis (Fig. 3A,B). This correlation persists even in cases where divisions are not oriented along the P-D axis (Fig. 3B; Supplemental Figs. S2-S4). These results indicate that the geometry of apical cell-cell junctions determines the orientation of cell division.
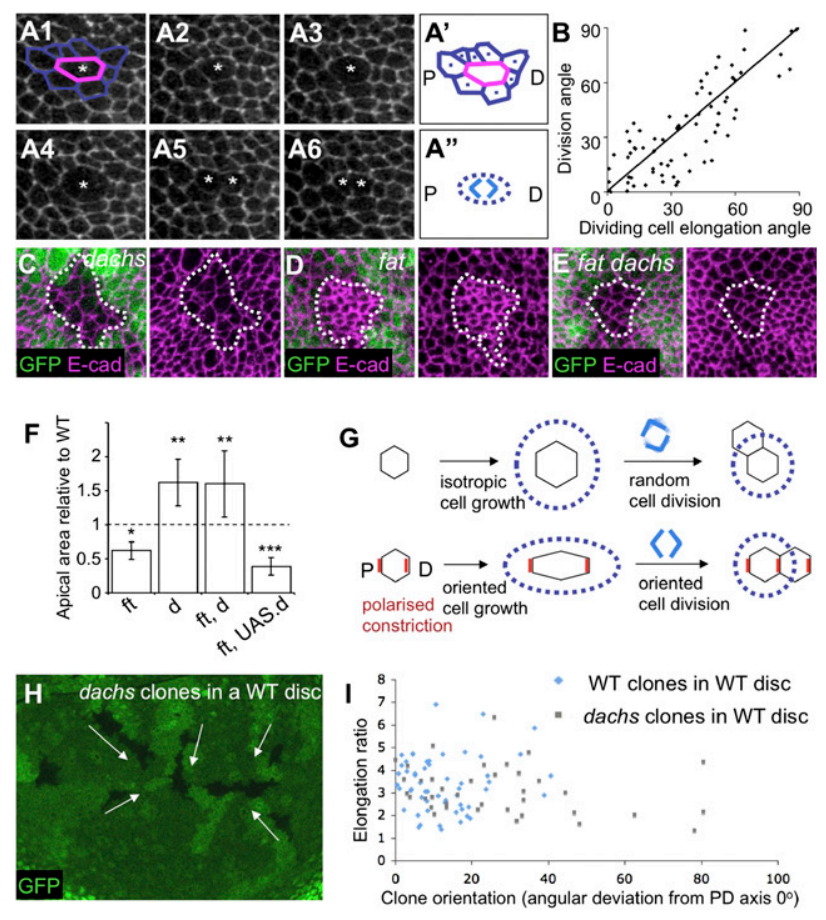

Figure 3. Dachs promotes constriction of apical cell-cell junctions to control cell shape, which may orient the mitotic spindle. (A1-6) Frames (5 min apart) from a live-imaged cell division in the wing pouch. $\left(A^{\prime}\right)$ Cell junction diagram with geometric centers of neighboring cells marked. $\left(A^{\prime \prime}\right)$ Ellipse drawn through vertices of dividing cells (elongation ratio $=1.45 \pm 0.31, n=95$ ) predicts the orientation of the mitotic spindle (light blue) and the plane of division. $(B)$ Correlation between a dividing cell's elongation angle and orientation of cell division, both relative to the $\mathrm{P}-\mathrm{D}$ axis. Pearson's correlation coefficient, $r=0.81(n=72)$. (C) dachs mutant clones (GFP-negative) dilate the apical surface (marked by E-cad). (D) Ectopic localization of Dachs around the circumference of apical junctions in a fat mutant clone (GFP-negative) constricts the apical surface. (E) In fat dachs doublemutant clones (GFP-negative) apical junctions are not constricted but are dilated like dachs clones. (F) Quantification of $C-E$ (Supplemental Table S1; Supplemental Fig. S7). Error bars, standard deviation. ( $\left.{ }^{\star}\right)$ fat mutant cells are apically more constricted than wild-type (WT) $(P<$ $0.001) .\left(^{\star \star}\right)$ dachs and fat/dachs mutants are apically more dilated than wild type $(P<0.001)$ but indifferent from each other. $\left(^{\star \star \star}\right)$ Overexpression of Dachs enhances constriction of fat mutant cells $(P<0.001)$. (G) A model of cell growth, shape, and divisions in an isotropic manner (top) and when planar-polarized by Dachs-mediated tension (bottom). (H) Oriented growth of dachs mutant clones is rescued when they are surrounded by wild-type (WT) cells. (I) Quantification of $H$ versus wild-type clones. The elongation ratio of dachs clones surrounded by wild-type (WT) cells is $3.22 \pm 1.07(n=38)$, indifferent from wild-type clones $(P=0.3)$ (Fig. 1H).
To test whether Dachs controls cell shape at the apical surface, we examined clones of dachs or fat mutant cells in an otherwise wild-type disc. Cells mutant for dachs are, on average, $60 \%$ more dilated in their apical surface area than wild type (Fig. 3C,F). Cells mutant for fat are, on average, $40 \%$ more constricted than neighboring wild-type cells (Fig. 3D,F). When fat mutant cells are also mutant for dachs, their apical surface area reverts to that of single dachs mutants (Fig. 3E,F). Overexpression of Dachs enhances constriction of fat mutant cells (Fig. 3F). These results show that Dachs exerts a contractile force on apical cell junctions. Since Dachs is normally planarpolarized, it would be predicted to constrict cell-cell junctions at the distal end of each cell and the proximal end of its neighbor (Fig. 3G). Measurement of tension indicates that distal (and proximal) cell-cell junctions are under more tension than others (Supplemental Fig. S5). Consequently, the cell must grow by lengthening its other cell-cell junctions, resulting in cell shape elongation in the $\mathrm{P}-\mathrm{D}$ axis prior to division, which then orients the mitotic spindle (Fig. 3G). This force-driven model is supported by the fact that clones of cells mutant for dachs show normally oriented growth when surrounded by wild-type cells that are capable of nonautonomously exerting force on the mutant clone (Fig. $3 \mathrm{H}, \mathrm{I}$; Mao et al. 2006). Thus, Dachs appears to act by exerting mechanical force because its loss can be compensated by restoring these forces.

To further test our proposal, we built a computational model of a growing epithelial tissue adapted from a previous model (see the Supplemental Material; Supplemental Figs. S8-S10; Farhadifar et al. 2007). We found that, compared with isometric apical tension (Fig. 4A; Supplemental Movie S2), polarized apical tension is sufficient to orient cell divisions and tissue growth (Fig. 4B; Supplemental Movie S3). As in vivo (Fig. 1; Baena-Lopez et al. 2005), cell divisions are only imperfectly correlated with the P-D axis in our model (Supplemental Fig. S10), and this emergent behavior is in fact important to generate the type of clone shapes observed in the developing wing (Fig. 4B,D). If cell divisions are forcibly oriented in the P-D axis, abnormal clone shapes result (Fig. 4C,D; Supplemental Movie S4) and the link between cell shape and orientation of division is broken (Fig. 5A-C), indicating that Dachs is unlikely to directly orient the spindle in vivo. Our model therefore supports the idea that Dachs indirectly orients the mitotic spindle by polarizing apical constriction to promote cell shape elongation in the $\mathrm{P}-\mathrm{D}$ axis.

Our model also explains an apparent paradox: Despite the presence of planar-polarized Dachs, the apical geometry of most wing cells is not elongated in the P-D axis. Indeed, elongation of cells in the P-D axis is commonly observed only in those cells about to undergo division. Our simulations show that this is because elongated cells divide to produce daughter cells of more rounded or random shapes. The polarized tension exerted by Dachs is of moderate strength, and hence takes time to promote elongation in the $\mathrm{P}-\mathrm{D}$ axis. Thus, only older cells ready to divide once more tend to show elongated forms. Furthermore, some cells never manage to elongate along the P-D axis, explaining why not all cell divisions occur in the P-D axis. Our model shows how this complex behavior of cells in a tissue emerges simply from the mechanical properties of a dividing epithelium. 


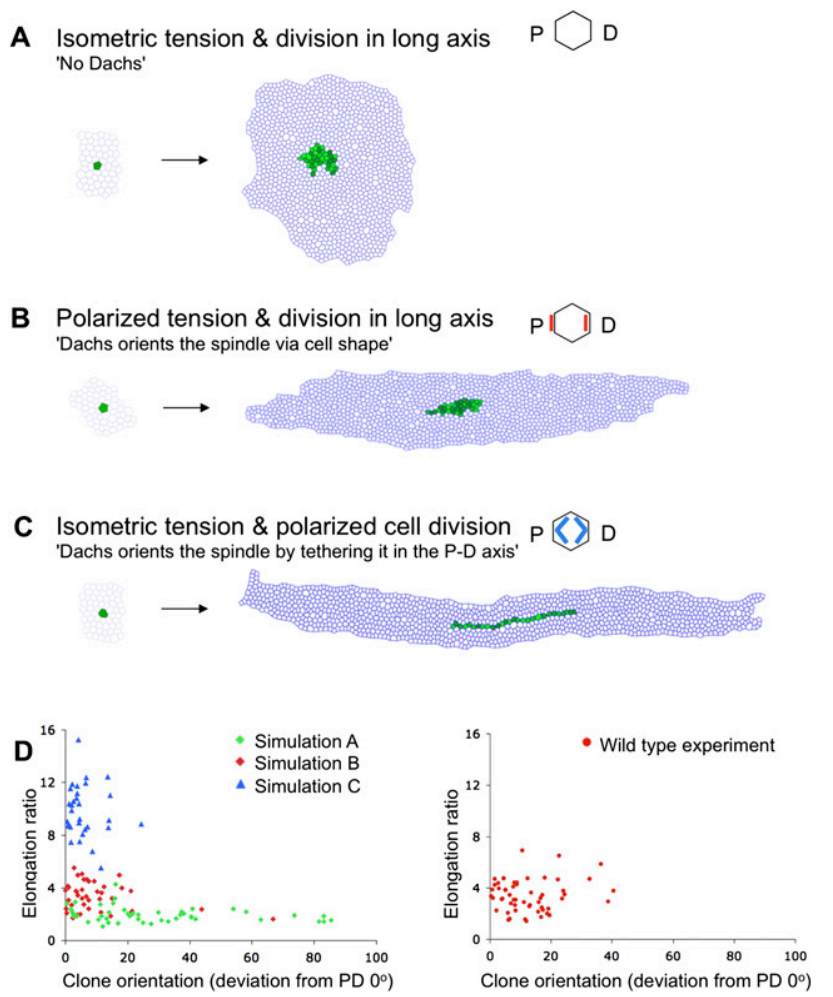

Figure 4. Computer simulations show that polarized tension is sufficient to orient cell divisions and tissue growth. See Supplemental Movies S2-S4. For detailed parameter explorations, see Supplemental Figures S6 and S7. $(A)$ Isometric tension at junctions leads to isometric clone shapes. $(B)$ Polarized tension at junctions orients most cell divisions in the $\mathrm{P}-\mathrm{D}$ axis, producing clone shapes and $\mathrm{P}-\mathrm{D}$ orientation similar to in vivo clones. $(C)$ Isometric tension with cell divisions forcibly oriented in the P-D axis produces clone shapes that are more elongated and P-D-aligned than in vivo clones. $(D)$ Quantification of simulated clone shapes compared with wild-type in vivo clones.

It is interesting to compare the function of the Dachs myosin in the proliferating wing epithelium with the function of myosin-II in convergent extension movements of the quiescent embryonic epithelium. Both myosins appear to generate anisotropic junctional tension, but at different strengths. In embryos, myosin-II is planar-polarized at gastrulation and exerts very strong tension, collapsing cell-cell junctions entirely such that cells rearrange their positions and intercalate. In the wing disc, Dachs appears much weaker than myosin-II because convergent extension movements are not obvious in live-imaged wing disc epithelia. Thus, orientation of cell divisions appears to require much more subtle polarization of cell tension than convergent extension, and this may explain why an atypical myosin, rather than myosinII, orients cell divisions. It will be interesting to investigate the role of Dachs in other oriented cell division scenarios, such as wound healing (Li et al. 2009).

In conclusion, planar polarization of Dachs links longrange gradients of secreted morphogens-known to be the fundamental organizers of tissue pattern, size, and shapewith the orientation of cell divisions and tissue growth during development. The mechanism we propose-a gradient directing the planar polarization of a molecule, which then orients cell behavior-may prove to be a widespread mechanism for organizing tissue development.

\section{Materials and methods}

\section{Generation of marked clones}

Clones marked by absence of ubiquitin.GFP expression were induced by FLP/FRT-mediated mitotic recombination with an FRT4O ubiquitin.GFP chromosome (Bloomington). FLP activity (recombination) was induced $72-96 \mathrm{~h}$ after egg laying by a $60-\mathrm{min}$ heat shock at $37^{\circ} \mathrm{C}$. Clones marked by expression of actin.lacZ were generated by FLP/FRT-mediated in cis recombination to remove a CD2 stop cassette from the actin.FRT.CD2. FRT.1acZ transgene (Bloomington). FLP activity was induced $48-72 \mathrm{~h}$ after egg laying by a $10-\mathrm{min}$ heat shock. Clone shapes were outlined and overlaid following rotational alignment along the P-D axis with Microsoft Powerpoint software.

\section{Drosophila genotypes}

The following Drosophila genotypes were used: whs.Flp/+; FRT40/FRT40 ubiquitin.GFP (Fig. 1C); yw hs.Flp/+; UAS.Dachs-V5/+; actin.FRT.CD2.FRT Gal4, UAS.GFP /+ (Fig. 1D); yw hs.Flp/+; actin.FRT.CD2.FRT lacZ/+ (Fig. 1E); yw hs.Flp/+; dachs ${ }^{G C 13} /$ dachs $^{G C 13}$; actin.FRT.CD2.FRT lacZ/+ (Fig. 1F); yw hs.Flp/+; fat ${ }^{G R V} /$ fat $^{8}$; actin.FRT.CD2.FRT lacZ/+ (Fig. 1G); w (Figs. 1I,L, 2E); w; dachs ${ }^{G C 13} /$ dachs $^{G C 13}$ (Fig. 1J); ; $^{\text {fat }}{ }^{G R V} /$ fat $^{8}$ (Fig. 1K); w; dachs $s^{G C 13}$ / dachs $^{G C 13}\left(\right.$ Fig. 1M); w; fat $^{\text {GRV } / f a t}{ }^{8}$ (Fig. 1N); yw hs.Flp/+; actin.FRT.CD2.FRT lacZ/+ (Fig. 2B, top); w; UAS.Dachs-V5; dpp.Gal4 UAS.GFP (Fig. 2B, bottom); yw hs.Flp/+; UAS.Ds/+; dpp.Gal4 UAS.GFP/actin.FRT.CD2.FRT lacZ (Fig. 2C, top); w; UAS.Dachs-V5/UAS.Ds; dpp.Gal4 UAS.GFP (Fig. 2C, bottom);
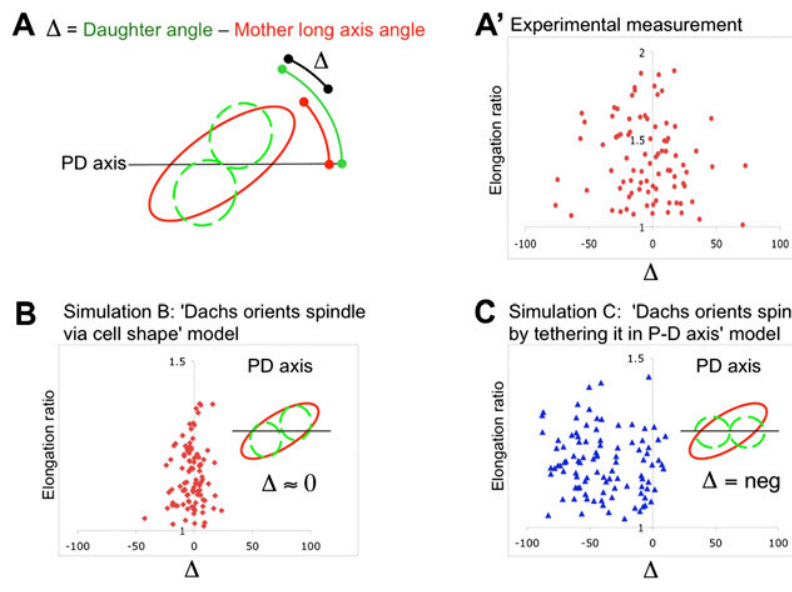

C Simulation C: 'Dachs orients spindle
by tethering it in P-D axis' model by tethering it in P-D axis' mode

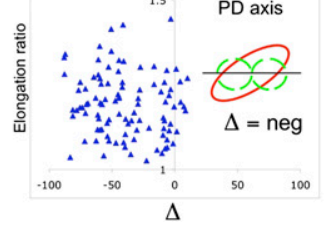

Figure 5. Evidence against Dachs directly orienting the mitotic spindle by tethering it in the P-D axis. $(A)$ The difference $(\Delta)$ between the angle of daughter cells (relative to $\mathrm{P}-\mathrm{D}=0$ ) and the long axis of their mother cell (relative to $\mathrm{P}-\mathrm{D}=0$ ) is near 0 if orientation of cell shape determines the orientation of division. The difference $(\Delta)$ is negative if the daughter cells are more P-D-aligned than their mother. According to the "cell shape orients the spindle" model, the difference $(\Delta)$ should be centered around 0 . According to the "Dachs directly orients the spindle" model, the difference $(\Delta)$ should be negative, because divisions would be expected to be biased along the P-D axis regardless of the shape of the mother cell. $\left(A^{\prime}\right)$ Analysis of in vivo cell division data from live imaging. The spread of the difference $(\Delta)$ is symmetric around the P-D axis (0). For rounder cells (elongation ratio close to 1), the long axis is less defined, thus giving a wider spread of difference $(\Delta)$. (B) In simulation B (polarized tension and division in long axis), cell shape orients the mitotic spindle and the difference $(\Delta)$ is symmetric, with a wider spread of difference $(\Delta)$ for rounder cells, as in vivo. $(C)$ In simulation $\mathrm{C}$ (isometric tension and polarized cell division), spindles are tethered in the P-D axis (by Dachs) and the difference $(\Delta)$ is no longer symmetric and is negative. There is also no correlation between the elongation ratio and spread of the difference $(\Delta)$. This remains true even when the spindle-tethering mechanism is made imprecise enough to produce the correct clone shape (not shown). These results indicate that, in vivo, Dachs does not directly control spindle orientation. 
yw hs.Flp/+; dpp.Gal4 UAS.GFP/+; UAS.Ds/+ (Fig. 2F'); omb.Gal4/+; UAS.DS/+ (Fig. 2G'); W; Armadillo-GFP (Fig. 3A); yw hs.Flp/+; FRT4O dachs ${ }^{G C 13} /$ FRT40 ubiquitin.GFP (Fig. 3C); yw hs.Flp/+; FRT40 fat ${ }^{8} / F R T 40$ ubiquitin.GFP (Fig. 3D); yw hs.Flp/+; FRT40 fat dachs $^{\text {GC13 } / F R T 40 ~}$ ubiquitin.GFP (Fig. 3E).

\section{Immunostaining}

Discs were fixed for $30 \mathrm{~min}$ in $4 \%$ paraformaldehyde in PBS, washed with PBT (PBS, 0.3\% Triton X-100), blocked with PBT + 0.1\% BSA, and stained with primary and fluorescently conjugated secondary antibodies (Molecular Probes and Jackson ImmunoResearch) with additional PBT washes. The following primary antibodies were used: rabbit anti-V5 (Abcam), mouse anti-Wingless, mouse anti-Engrailed, rat anti-E-cad, mouse antiTubulin (all from Developmental Studies Hybridoma Bank), rabbit antiPH3 (Upstate Biotechnologies), rabbit anti-ßgal (Cappel).

\section{Fixed-sample imaging}

Fluorescent imaging of fixed samples was performed with a Leica SP5 laser-scanning confocal microscope. Bright-field imaging of adult tissues was performed with a Zeiss Axioplan microscope. Images were processed in Adobe Photoshop.

\section{Live imaging}

Live imaging of ex vivo cultured wing discs was performed with a Perkin Elmer Spinning Disc microscope. Discs were cultured in a $35-\mathrm{mm}$ Fluorodish with Shields and Sang M3 media containing $2 \%$ fetal bovine serum, $10 \mathrm{ug} / \mathrm{mL}$ streptomycin/penicillin, $10 \mathrm{mU} / \mathrm{L}$ insulin, and $2.5 \%$ methyl cellulose (Aldaz et al. 2010). Z-stacks were taken at 1- $\mu \mathrm{m}$ intervals, with total thickness of 20-30 $\mu \mathrm{m}$. $Z$-stacks were scanned at 2-min intervals for up to $5 \mathrm{~h}$. Images were projected and time points were collated in Improvision Volocity software.

\section{Statistical analysis of elongation and orientation of cell divisions and clone shapes}

Statistical analyses of the orientation of clone shapes and cell divisions were performed with software purpose-built using the OpenCV library, an open-source image analysis library written in C (see Supplemental Fig. S6). The software used the least-square fitting algorithm provided in OpenCV (cvFitEllipse2) to fit ellipses to the shape of entire clones,or the dividing cell (just before mitosis) and the position of daughter cells, as indicated by the user by selecting certain points outlining the clone and cells. The elongation ratios, centers, and main axis of these ellipses were then used to calculate angles and for further statistical analysis. $P$-values were calculated with a Wilcox nonparametric test.

\section{Systematic definition of the $P-D$ axis}

To define the P-D axis in the wing pouch, we used a mathematical approximation that agrees with the experimentally observed orientation of wild-type clones: The distal-most segment of the wing pouch was defined as a line along the dorsal-ventral (D-V) boundary between two lateral points, determined by the user (Supplemental Fig. S6, white arrows). The theoretical (ideal) P-D orientation for a particular position (e.g., of the center of a cell, spindle, or clone) was calculated by projecting that point on the distal-most segment and moving one-third of the way from the projection point toward the center of the distal-most segment (Supplemental Fig. S6B).

\section{Apical cell surface area quantification}

Cell area measurements and cell counts were performed with software purpose-built using the OpenCV library, an open-source image analysis library written in C (see Supplemental Fig. S7). Since the apical surface area of cells in the wing disc varies depending on its position from the center of the disc, mutant cell surface areas must be compared with their immediate wild-type neighbors that are in the same relative positions in the wing disc. Dividing the area of the clone by number of cells in this clonal region gave the average area of cells in that clone. Dividing the average mutant apical cell area by the average wild-type apical cell area gave the ratio of mutant to wild-type area. The ratios for the apical area of each mutant clone were then averaged, and the mean and standard deviation were calculated. $P$-values were calculated with a Wilcox nonparametric test. See Supplemental Table S1 for further details.

\section{Acknowledgments}

We thank Ken Irvine for fly stocks, Silvia Aldaz for advice on wing disc culturing, and Frederic Bollet-Quivogne for help with laser ablations. This work was supported by Cancer Research UK. Y.M. is supported by a Medical Research Council (MRC) Biomedical Informatics Fellowship (G0802456).

\section{References}

Aldaz S, Escudero LM, Freeman M. 2010. Live imaging of Drosophila imaginal disc development. Proc Nat1 Acad Sci 107: 14217-14222.

Baena-Lopez LA, Baonza A, Garcia-Bellido A. 2005. The orientation of cell divisions determines the shape of Drosophila organs. Curr Biol 15: $1640-1644$.

Bennett FC, Harvey KF. 2006. Fat cadherin modulates organ size in Drosophila via the Salvador/Warts/Hippo signaling pathway. Curr Biol 16: 2101-2110.

Cho E, Irvine KD. 2004. Action of fat, four-jointed, dachsous and dachs in distal-to-proximal wing signaling. Development 131: 4489-4500.

Cho E, Feng Y, Rauskolb C, Maitra S, Fehon R, Irvine KD. 2006. Delineation of a Fat tumor suppressor pathway. Nat Genet 38: 1142-1150.

Clark HF, Brentrup D, Schneitz K, Bieber A, Goodman C, Noll M. 1995. Dachsous encodes a member of the cadherin superfamily that controls imaginal disc morphogenesis in Drosophila. Genes Dev 9: 1530-1542.

Farhadifar R, Roper JC, Aigouy B, Eaton S, Julicher F. 2007. The influence of cell mechanics, cell-cell interactions, and proliferation on epithelial packing. Curr Biol 17: 2095-2104.

Feng Y, Irvine KD. 2007. Fat and expanded act in parallel to regulate growth through warts. Proc Nat1 Acad Sci 104: 20362-20367.

Hafen E. 2004. Interplay between growth factor and nutrient signaling: Lessons from Drosophila TOR. Curr Top Microbiol Immunol 279: 153-167.

Lawrence PA, Struhl G. 1996. Morphogens, compartments, and pattern: Lessons from Drosophila? Cell 85: 951-961.

Lecuit T, Le Goff L. 2007. Orchestrating size and shape during morphogenesis. Nature 450: 189-192.

Li W, Kale A, Baker NE. 2009. Oriented cell division as a response to cell death and cell competition. Curr Biol 19: 1821-1826.

Lu B, Roegiers F, Jan LY, Jan YN. 2001. Adherens junctions inhibit asymmetric division in the Drosophila epithelium. Nature 409: 522525.

Mahoney PA, Weber U, Onofrechuk P, Biessmann H, Bryant PJ, Goodman CS. 1991. The fat tumor suppressor gene in Drosophila encodes a novel member of the cadherin gene superfamily. Cell 67: 853-868.

Mao Y, Rauskolb C, Cho E, Hu WL, Hayter H, Minihan G, Katz FN, Irvine KD. 2006. Dachs: An unconventional myosin that functions downstream of Fat to regulate growth, affinity and gene expression in Drosophila. Development 133: 2539-2551.

Matakatsu H, Blair SS. 2004. Interactions between Fat and Dachsous and the regulation of planar cell polarity in the Drosophila wing. Development 131: 3785-3794.

Pan D. 2007. Hippo signaling in organ size control. Genes Dev 21: 886897.

Rogulja D, Rauskolb C, Irvine KD. 2008. Morphogen control of wing growth through the Fat signaling pathway. Dev Cell 15: 309-321.

Silva E, Tsatskis Y, Gardano L, Tapon N, McNeill H. 2006. The tumorsuppressor gene fat controls tissue growth upstream of expanded in the hippo signaling pathway. Curr Biol 16: 2081-2089.

Simon MA. 2004. Planar cell polarity in the Drosophila eye is directed by graded Four-jointed and Dachsous expression. Development 131: 6175-6184. 
Mao et al.

Thery M, Jimenez-Dalmaroni A, Racine V, Bornens M, Julicher F. 2007. Experimental and theoretical study of mitotic spindle orientation. Nature 447: 493-496.

Vogel SK, Raabe I, Dereli A, Maghelli N, Tolic-Norrelykke I. 2007. Interphase microtubules determine the initial alignment of the mitotic spindle. Curr Biol 17: 438-444.

Willecke M, Hamaratoglu F, Kango-Singh M, Udan R, Chen CL, Tao C, Zhang X, Halder G. 2006. The fat cadherin acts through the hippo tumor-suppressor pathway to regulate tissue size. Curr Biol 16: 20902100.

Willecke M, Hamaratoglu F, Sansores-Garcia L, Tao C, Halder G. 2008. Boundaries of Dachsous Cadherin activity modulate the Hippo signaling pathway to induce cell proliferation. Proc Natl Acad Sci 105: 14897-14902. 


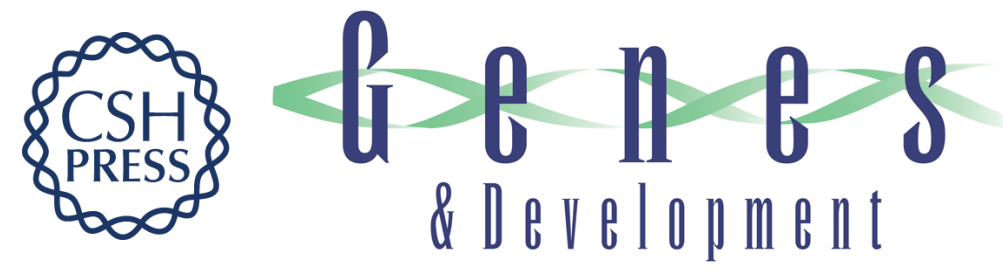

\title{
Planar polarization of the atypical myosin Dachs orients cell divisions in Drosophila
}

\author{
Yanlan Mao, Alexander L. Tournier, Paul A. Bates, et al.
}

Genes Dev. 2011, 25:

Access the most recent version at doi:10.1101/gad.610511

\section{Supplemental http://genesdev.cshlp.org/content/suppl/2011/01/18/25.2.131.DC1 Material}

References This article cites 24 articles, 9 of which can be accessed free at: http://genesdev.cshlp.org/content/25/2/131.full.html\#ref-list-1

\section{License}

Email Alerting

Receive free email alerts when new articles cite this article - sign up in the box at the top Service 